\title{
Sanierung, Revitalisierung und Modernisierung von Kleinwasserkraftwerken
}

\begin{abstract}
Zusammenfassung: Die KELAG-Kärntner Elektrizitäts-Aktiengesellschaft betreibt über 60 Kraftwerke, eine große Anzahl davon sind Kleinwasserkraftwerke. Die ältesten sind über 100 Jahre in Betrieb. In den vergangenen 20 Jahren wurden umfangreiche Sanierungen, Revitalisierungen und Modernisierungen an diesen Kraftwerken durchgeführt, wobei diesen Tätigkeiten unterschiedlichste Zielsetzungen voraus gingen. Neben baulichen Ertüchtigungen und Anpassungen an ökologische Erfordernisse wurden vielfach Modernisierungen durch Automatisierung, Fernüberwachung/Fernsteuerung und Leittechnikverbesserung sowie sicherheitstechnische Anpassungen an den Stand der Technik umgesetzt. In einigen Fällen konnten nennenswerte Wirkungsgradverbesserungen erzielt werden. Im Folgenden werden ausgewählte Beispiele im Detail beschrieben.
\end{abstract}

Upgrading, revitalising and modernising small power stations

Summary: KELAG Kärntner ElektrizitätsAktiengesellschaft in Carinthia, Austria, runs more than 60 power stations, of which a large proportion are small. The oldest among them have been working for more than 100 years. The past two decades have seen comprehensive upgrading, revitalising and modernising schemes implemented at these stations, aiming at a variety of goals, including structural enhancements and adjustments to answer ecological requirements, modernisation of plant by automation, installation of telemonitoring/telecontrol systems, improvement of process control as well as upgrading safety systems to meet state-of-the-art requirements. In some cases, it has been possible to achieve considerable efficiency improvement. This article describes selected projects in detail.

\section{Kraftwerk Kamering}

Der Maschinensatz des Kraftwerkes Kamering, bestehend aus einer Francis-Turbine mit vertikaler Welle und einem 10 MVA-

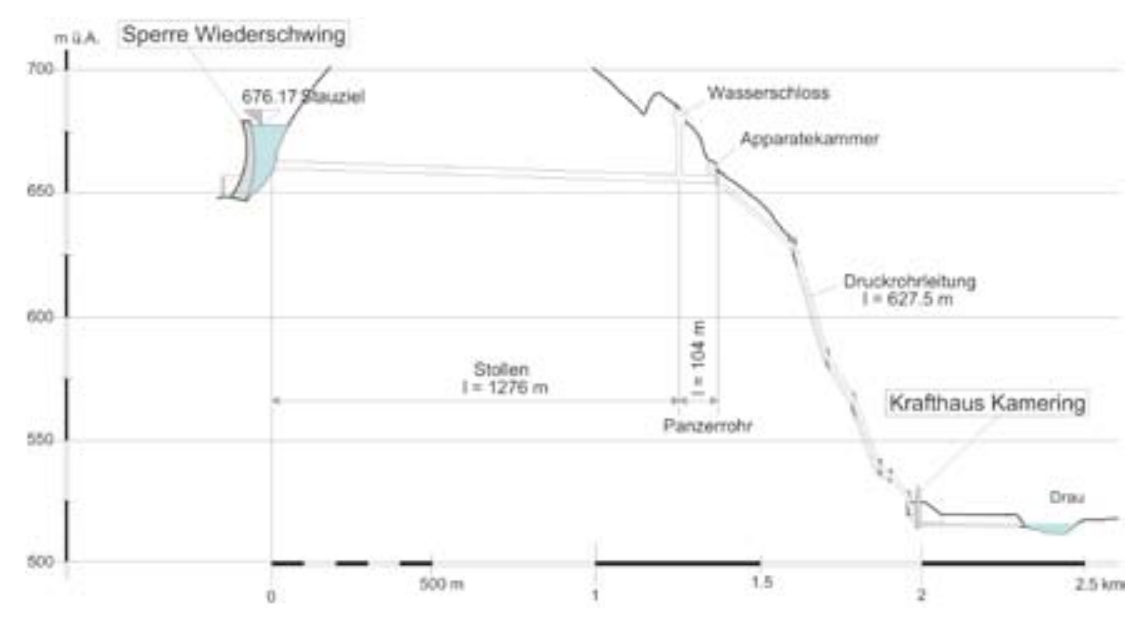

Abb. 1: Kraftwerk Kamering - Triebwasserweg/Übersichtslängenprofil

Synchrongenerator, wurde 1952 in Betrieb genommen. Im Jahre 1996, nach 44 Betriebsjahren, in welchen $1450 \mathrm{GWh}$ erzeugt wurden, erreichte vor allem der Generator das Ende seiner technischen Lebensdauer. Es wurden daher ein Generatortausch und eine Generalsanierung der Francis-Turbine durchgeführt, wobei durch den Einsatz einer neuen Turbinenhydraulik eine wesentliche Wirkungsgradverbesserung erzielt werden konnte. Weiters wurden bauliche Maßnahmen zur Erhöhung der Sicherheit an der Sperre vorgenommen, auf welche in diesem Bericht nicht eingegangen wird.

\subsection{Anlagenbeschreibung}

Das Kraftwerk Kamering liegt in Mittelkärnten im Drautal, ca. $10 \mathrm{~km}$ flussabwärts der Stadt Spittal/Drau. Es nützt das Wasserdargebot eines Einzugsgebietes von $153 \mathrm{~km}^{2}$, das einerseits in einem künstlichen Wochenspeicher aufgefangen wird, andererseits wird der Weißensee, der höchstgelegene Badesee Kärntens, innerhalb seiner natürlichen Spiegelschwankungen als Fernspeicher verwendet. Vom Wochenspeicher Wiederschwing, dessen Talabschluss eine doppelt gekrümmte Gewölbemauer bildet, wird das Triebwasser über einen $1.400 \mathrm{~m}$ langen Stollen und danach über eine $630 \mathrm{~m}$ lange Rohrleitung zum Kraftwerk geleitet (Abbildung 1). Das
Triebwasser wird über einen vertikalen Maschinensatz, im Wesentlichen bestehend aus einer Francis-Turbine und einem 10 MVA-Synchrongenerator, abgearbeitet. Der Abfluss vom Krafthaus erfolgt über einen ca. $350 \mathrm{~m}$ langen Unterwasserkanal in die Drau, wobei die Einmündung direkt in den Stauraum des Kraftwerks Paternion der ehemaligen Draukraftwerke (heute AHP) erfolgt.

\subsubsection{Wesentliche Kraftwerksdaten}

Einzugsgebiete:

$153 \mathrm{~km}^{2}$

Speicher Wiederschwing - Nutzinhalt:

1,15 Mio $^{3}$

Druckstollen - Durchmesser/Länge:

$2,6 \mathrm{~m} / 1.380 \mathrm{~m}$

Druckrohrleitung - Durchmesser/Länge:

$1,8 \div 1,5 \mathrm{~m} / 627,5 \mathrm{~m}$

Rohfallhöhe:

$161,2 \mathrm{~m}$

Ausbauwassermenge:

$7 \mathrm{~m}^{3} / \mathrm{sec}$.

Nenndrehzahl/Turbinennennleistung alt: $600 \mathrm{UpM} / 8,95 \mathrm{MW}$

Jahreserzeugung alt:

$31,5 \mathrm{GWh} / \mathrm{Jahr}$

Nenndrehzahl/Turbinennennleistung neu: 750 UpM/9,9 MW

Jahreserzeugung neu:

33,8 GWh/Jahr 


\subsection{Erneuerungserfordernisse und durchgeführte Maßnahmen}

\subsubsection{Generator}

Im Kraftwerk Kamering war bis zum Umbau ein vertikalachsigerer, 10 MVA-Synchrongenerator mit einem oberen kombinierten Trag- und Führungslager und einem unteren Führungslager eingesetzt. Die nicht für das Kraftwerk Kamering konzipierte Maschine wurde vor dem 2. Weltkrieg gefertigt und konnte auf Grund der Kriegswirnisse nicht mehr nach Südamerika ausgeliefert werden. Der in der Folge von der KELAG günstig erworbene Generator wies zwar wie beabsichtigt die zu installierende Maschinenleistung auf, war aber für eine Drehzahl von nur 600 UpM konzipiert und mit dieser zu betreiben.

Durch Auslegung der Turbinenhydraulik auf die vom Generator vorgegebene niedrigere Drehzahl - den hydraulischen Verhältnissen entsprechend wäre eine Drehzahl von 750 UpM optimal gewesen wurde dieser Mangel so gut wie möglich ausgeglichen.

Innerhalb der 4 Jahrzehnte erfolgten am Generator zwei Großrevisionen, welche mit Ausnahme einer Spurlagererneuerung, die auf eine fehlerhafte Bedienung zurückzuführen war, im wesentlichen der Reinigung bzw. Nachverfestigung von Blechpaketen und Wicklungen dienten. Seit der letzten Generalüberholung im Jahre 1980 wurde in periodischen Intervallen Diagnosemessungen durchgeführt, die eine laufende Verschlechterung des Isolationszustandes der Statorwicklung ergaben. Im Jahr 1994 musste festgestellt werden, dass der Generator nach damals ca. 42-jähriger Betriebsdauer das Ende seiner technischen Lebensdauer erreicht hat.

Es wurde daher die Entscheidung gefällt, den Generator im Jahre 1996 zur Gänze gegen einen neuen auszutauschen und die Leistung auf 11 MVA sowie die Drehzahl - entsprechend einer optimalen Turbinenhydraulik - auf $750 \mathrm{UpM} \mathrm{zu}$ erhöhen.

Die Aufstellung des Generators erfolgt auf den ursprünglichen Montagefüßen. Das einteilige Statorgehäuse stellt eine aus Walzstahl bestehende Schweißkonstruktion dar, die durch Rippen und Ringspanten derart versteift ist, dass sich die gewünschte Schwingungs- und Biegesteifigkeit ergibt.

Der Läufer besteht aus einer durchgehend geschmiedeten Welle, der aktive

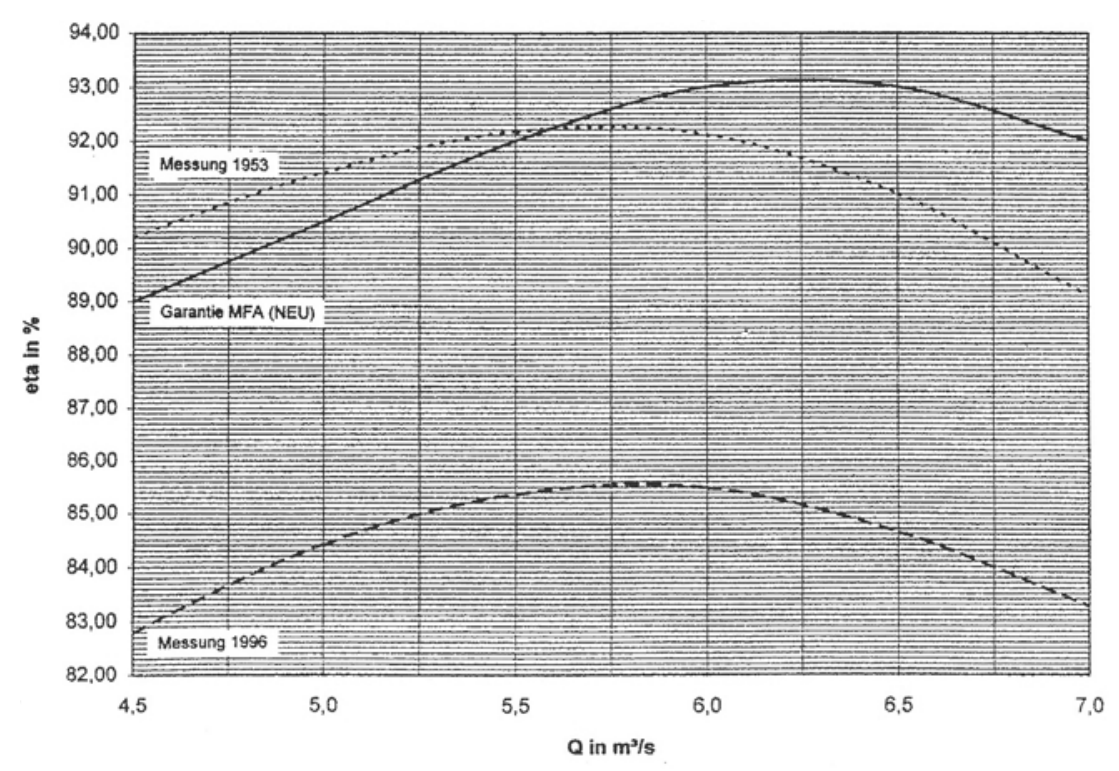

Abb. 2: Kraftwerk Kamering Turbinenwirkungsgrad

Teil aus einer Kammpolkonstruktion, welche die Erreger- und die Dämpferwicklung trägt. Darüber hinaus erhielt der Generator eine Wechselstromerregermaschine mit umlaufenden Dioden.

Die Segmentlager des Generators weisen Ölsumpfschmierungen auf und sind ohne Lagerölheizungen ausgeführt. Die Kühlung des Lageröls erfolgt in externen Ölkühlern, wobei die Zirkulation des Öls mittels in den Führungslagersegmenten integrierter Öltaschen erreicht wird. Die Lager werden mit Ausnahme der Lagerentlastungspumpe beim Spurlager ohne Hilfsaggregate (Anfahrpumpen, Heizungen) betrieben.

Das Kuppeln der Generator- mit der Turbinenwelle erfolgt über 10 hydraulisch vorgespannte Passschrauben. Um eine möglichst genaue Übereinstimmung der getrennt hergestellten Passlöcher zu erreichen, wurde das Lochbild des Generatorwellenflansches mittels Schablone auf die Turbinenwelle übertragen. Die dabei erzielte Übertragungsgenauigkeit betrug ca. 0,01 mm. Durch geringfügiges Nachreiben der Schraubenlöcher auf der Baustelle war eine rasche Montage möglich.

\subsubsection{Maschinenleittechnik}

Die bis zum Umbau eingesetzte, in klassischer Relaistechnik ausgeführte Maschinensteuerung ermöglichte, auch bedingt durch das Fehlen einer Hochdruckentlastungseinrichtung beim Spurlager, nur teilautomatisches Anfahren.

Nach dem Parallelschalten wurde durch Zugriff auf die Drehzahlverstellung (Wirkleistungsregelung) und den elektromechanischen Spannungsregler (Bildleistungsregelung) der Fernlastbetrieb aufgenommen.

Das Stillsetzen des Maschinensatzes konnte ebenfalls von der Ferne vorgenommen werden und erfolgt automatisch.

Neue Maschinenleittechnik:

Die neue Maschinenautomatik wurde in Schrankbauweise errichtet, wobei die Komponenten

- Maschinenanfahr- und Stillsetzautomatik (inkl. Synchronisiereinrichtung und Q-Regelung)

- mechanischer Schutz mit Geberüberwachung, Gefahrmeldung,

- Protokolliereinrichtung und Fernwirkankopplung

- und elektrischer Generatorschutz

in digitaler Technik ausgeführt und gemeinsam mit den Komponenten

- Hilfsbetriebesteuerschrank

- Maschinenleitstand (Mosaik)

- maschinenbezogene Eigenbedarfsverteilung

- und Erregungseinrichtung inkl. Spannungsregler

in der bestehenden Warte, angepasst an die bereits vorhanden gewesene Wartentafel, aufgestellt sind.

Über eine serielle Schnittstelle zur Fernwirkeinrichtung wird der Maschinensatz von der Energieleitzentrale der KELAG in Klagenfurt aus ferngesteuert betrieben. 


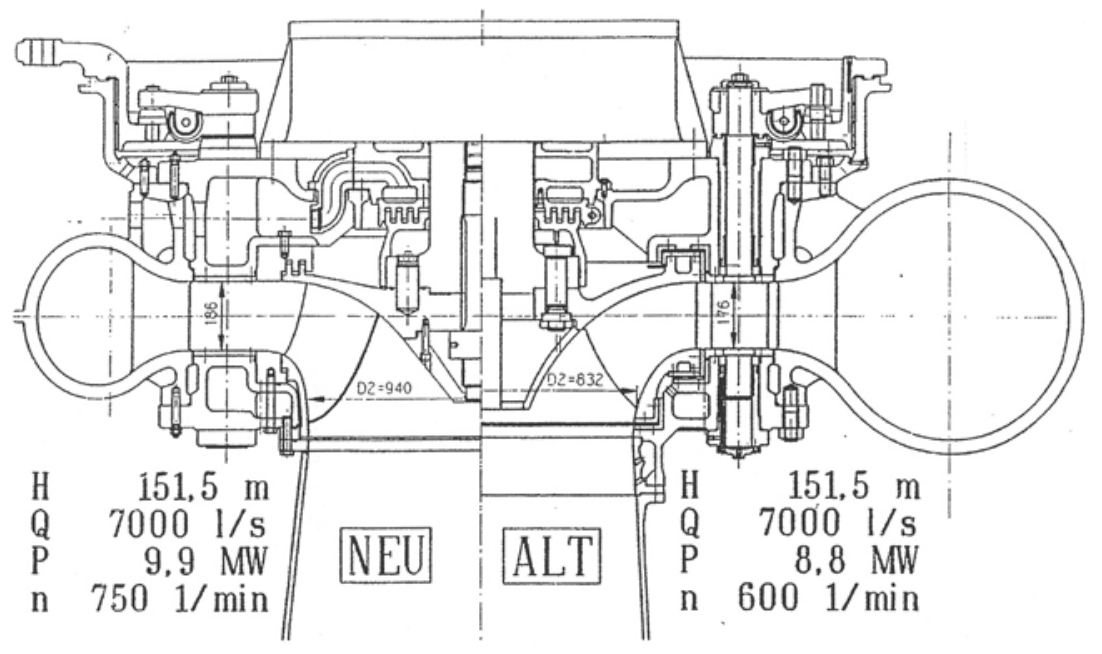

Abb. 3: Kraftwerk Kamering - Vergleich Hydraulik ALT - NEU

\subsubsection{Turbine}

Nach den 44 Betriebsjahren war auch die vertikale Francis-Turbine einer Generalrevision zu unterziehen. In Anbetracht der seinerzeit nicht optimal gewählten Drehzahl galt es auch Überlegungen anzustellen, inwieweit eine neue Turbinenhydraulik wirtschaftlich eingesetzt werden könnte.

Vom Lieferanten der Erstausrüstung der Maschinenfabrik Andritz (MFA) wurde bei Verwendung einer neuen Tubinenhydraulik z.T. deutlich höhere Wirkungsgrade im Vergleich zum alten Laufrad angegeben. Das neue Laufrad wurde im Auftrag der MFA von Sulzer Hydro mittels numerischer Strömungssimulationsrechnung entwickelt. Ziel war es, das Kavitationsverhalten den hydraulischen Verhältnissen angepasst zu verbessern, die Wirkungsgrade über den gesamten Betriebsbereich zu erreichen und eine ausgezeichnete Laufruhe zu gewährleisten. Zur Verifizierung der Wirkungsgrade des alten Laufrades wurde eine Messung nach der thermodynamischen Methode durchgeführt sowie auch beim neuen Laufrad zum Nachweis der Garantiewerte (Abbildung 2).

Die Berechnungen ergaben, dass als Maßnahme zur Umsetzung der neuen Turbinenhydraulik das Durchflussprofil (Spiralgehäuseaustritt) erhöht, ein neues Laufrad mit größerem Austrittdurchmesser sowie ein geänderter Saugrohrkonus eingebaut werden müssen (Abbildung 3). Weiters wurde die Drehzahl von $600 \mathrm{UpM}$ auf 750 UpM erhöht.

Unter Berücksichtigung des Massenträgheitsmomentes des Generators wurden von der MFA Berechnungen zur
Ermittlung der Drehzahl- und Druckanstiege durchgeführt. Dies für verschiedene Schließzeiten des Leitapparates und unter Einbeziehungen des Druckreglers sowie Weglassung desselben.

Nachdem die Bemessung der Druckrohrleitung seinerzeit für einen ausreichend hohen statischen Druck erfolgt war, wurde einvernehmlich festgelegt, dass der vorhandene Nebenauslass in $\mathrm{Zu}$ kunft stillgelegt wird und die erhöhten Drehzahlanstiege bei der Auslegung des Generators - insbesondere der Lager - zu berücksichtigen sind.

Weiters wurde an der Turbine nachfolgend angeführte Komponenten erneuert bzw. Umbauten durchgeführt:

- Neufertigung der Leitschaufeln aus GX5 CrNi 13.4 inkl. der Leitschaufellagerung und Dichtungen.

- Umbau des Turbinenlagers auf Selbstschmierung mit rotierender Ölwanne und Neuausgruß der Lagerschalen mit Weißmetall, wobei die Schwalbenschwanzverzahnung des Lagerkörpers erhalten blieb.

- Anpassung der stehenden Turbinenwelle an das neue Laufrad sowie an die Generatorwelle.

- Adaptierungsarbeiten am oberen und untern Turbinendeckel mit Einbau von neuen Leitschaufelschutzwänden und neuen Labyrinthringen aus Niro.

\subsubsection{Turbinenregler}

Als Turbinenregler fungierte ein mechanisch-hydraulischer Drehzahlregler, der mit einer Druckölanlage samt Windkessel ausgestattet war, welche zusätzlich auch der Steuerung des Druckreglers diente.
Während die Druckölanlage am Turbinenboden montiert war, wurde der Drehzahlregler in der um $7 \mathrm{~m}$ höher situierten Maschinenhalle aufgestellt, von wo aus der Antrieb über eine gleich lange vertikale Regulierwelle auf den Leitapparat erfolgte.

Der Drehzahlistwert wurde von einem am Läufer angebauten Pendelgenerator mittels elektronischer Welle übertragen.

Die Regelgenauigkeit erfüllte noch alle Erfordernisse. In Ermangelung jeglicher Ersatzteile sowie des Erneuerungsschrittes bei den sonstigen Anlagenkomponenten drängte sich jedoch auch die Erneuerung des Turbinenreglers auf. Weiters war das Hydraulikaggregat durch den Wegfall des Druckreglers nicht mehr in der gegebenen Größe erforderlich.

Zur Ausführung gelangte ein moderner Turbinenregler in digitaler Technik, bestehend aus

- dem digitalen Leitwerk,

- dem Hydraulikaggregat,

- dem Leitapparatantrieb und

- dem digitalen Turbinenregler.

Das digitale Leitwerk weist die Grundregelungsarten der Leistungs-, Öffnungs- und Drehzahlregelung auf. In Abstimmung mit dem hauptsächlich angewandtenBetriebsfallderLeistungsregelung wurde auch das Regelkonzept ausgelegt. Eine Inselregelung ist zwar hard- und softwaremäßig vorgesehen, jedoch nicht ausgeführt.

Ein mit separater Spannungsversorgung direkt auf das Regelventil wirkendes Handsteuergerät dient zur Verstellung des Leitapparates im Revisionsfalle bzw. ist auch bestens geeignet, bei ausgefallenem Turbinenregler einen Handnotbetrieb zu ermöglichen.

Das Hydraulikaggregat wurde für einen Betriebsdruck von 60 bar gegenüber zuvor 20 bar ausgelegt und konnte - auch durch Wegfall des Druckreglers - mit 120 Liter gegenüber zuvor 1.600 Liter Fassungsvermögen klein gehalten werden. Es ist mit sämtlichen üblichen Schutzeinrichtungen ausgestattet, wobei aber darauf geachtet wurde, nicht zwingend erforderliche Hilfseinrichtungen erst gar nicht vorzusehen. So konnte z. B. durch Auswahl eines dünnflüssigeren Öls die Reglerölheizung mit den erforderlichen Steuer- und Schutzeinrichtungen entfallen.

Der neue Leitapparatantrieb wirkt entsprechend der ursprünglichen Konzeption über ein Regulierherz und 2 Schubstangen auf den Leitapparat. Dabei wird die Aufseite des Servomotors mit Steueröl und die Schließseite mit Druckwasser aus der Triebwasserleitung beaufschlagt. 


\subsection{Demontage- und \\ Montagearbeiten sowie \\ Inbetriebsetzung}

Mit den Demontagearbeiten an Turbine und Generator (Abbildung 4) wurde Ende Mai 1996 begonnen. Erforderliche Vorarbeiten, sowie die Erneuerung des Maschinenhauskrans, der damals ebenfalls bereits ein Alter von 44 Jahre aufwies, wurde davor durchgeführt. Der demontierte Generator wurde durch die Lieferfirma entsorgt.

Die Turbinenteile wurden gereinigt, sandgestrahlt und einer Grundkonservierung im Kraftwerk Außerfragant der KELAG unterzogen und danach zur MFA geliefert, um dort die erforderlichen Adaptierungsarbeiten durchführen $\mathrm{zu}$ können.

Auf Grund von Setzungen des Bauwerks bereits unmittelbar nach der Kraftwerkserrichtung war es zu einer Schiefstellung des Turbinenspiralgehäuses im Ausmaß von ca. 1,5 mm gekommen. Diese Schiefstellung wurde durch das Abdrehen der Flanschflächen vor Ort korrigiert.

In weiterer Folge wurde das Durchflussprofil erhöht sowie eine Sandstrahlung und Konservierung des Spiralgehäuses vorgenommen. Das alte Saugrohr wurde herausgeschrämt, um den Einbau des etwas größeren neuen Saugrohres zu ermöglichen.

Nach den durchgeführten Adaptierungsarbeiten wurden die Turbinenteile Mitte Juli wieder von der MFA in das Kraftwerk Kamering retourniert und danach mit der Wiedermontage der Turbine begonnen.

Ab Ende Juli wurde auch die Generatormontage aufgenommen, wobei der ursprünglich vorgesehene Montageablauf gravierend geändert werden musste, da einerseits der Generator erst ab Anfang September sowie das Turbinenlaufrad erst ab Ende August für die Wiedermontage zur Verfügung standen. Die Generatormontage beschränkte sich somit vorerst auf den Stator und die peripheren Anlagenteile wie Ölversorgungen, Druckluftversorgung, etc. Die Montage der Turbine wurde vorerst mit einer Laufradattrappe vorgenommen und das Laufrad danach von unten, wobei der untere Turbinendeckel wieder entfernt werden musste, montiert.

$\mathrm{Ab}$ Anfang September erfolgt die Rotormontage mit nachfolgender Wellenfreigabe und Kuppeln der Turbinen- und Generatorwelle, sodass Ende September das 1. Drehen vorgenommen werden konnte.
Nach Durchführung der erforderlichen Inbetriebsetzungsarbeiten, wie Überprüfung der Schutz- und Gefahrmeldeeinrichtungen, Absolvierung der Automatikversuche, etc., konnte mit Mitte Oktober 1996 der Probebetrieb des Maschinensatzes aufgenommen werden.

Als wesentlich ist anzumerken, dass sämtliche Montagearbeiten an der Francis-Turbine durch KELAG-Personal durchgeführt wurden und lediglich die Montageaufsicht durch MFA beigestellt wurde.

Die Gesamtkosten für die Erneuerung des Maschinesatzes beliefen sich auf ca. $€ 3,5$ Mio.

\subsection{Fazit}

44 Betriebsjahre erforderten den Austausch des Generators im Kraftwerk Kamering, wobei gleichzeitig eine Generalsanierung der Francis-Turbine durchgeführt wurde.

Durch den Einsatz einer neuen Turbinenhydraulik ergab sich als angenehme Begleiterscheinung eine wesentliche Steigerung des Turbinenwirkungsgrades, die in weiterer Folge eine Mehrerzeugung von ca. 2 GWh pro Jahr bei einer Jahresdurchschnittserzeugung von bisher ca. $32 \mathrm{GWh}$ zu Folge hat.

Durch eine exakte Terminplanung gemeinsam mit den Lieferfirmen für $\mathrm{Ge}$ nerator und Turbine konnten die abzuwickelnden Demontage- und Montagearbeiten in einem Zeitraum von etwas mehr als 4 Monaten bewältigt werden. Dies ist auch deshalb bemerkenswert, weil es bei der Auslieferung maßgeblicher Anlagenkomponenten, wie Rotor und Turbinenlaufrad, Terminschwierigkeiten gab, die einen Mehraufwand an Montagearbeiten erforderten.

Darüber hinaus wurde zur Erhöhung der Betriebssicherheit und einer Empfehlung des Unterausschusses der Staubeckenkommission folgend, an der Gewölbesperre des Speichers Wiederschwind die Hochwasserentlastung und der Grundablass umgebaut.

\section{Kraftwerk Kaningbach}

\subsection{Ausgangssituation}

Das Kraftwerk Kaningbach weist mit seinen vier Maschinensätzen (2 Francis, je 1470 Kilowatt; 2 Pelton, je 590 Kilowatt) eine Gesamtnennleistung von 4,1 MW auf und erzielt ein Jahresregelarbeitsvermögen von 15,6 GWh. Die Kraftwerksanlage verfügt über zwei Bachfassungen und ist

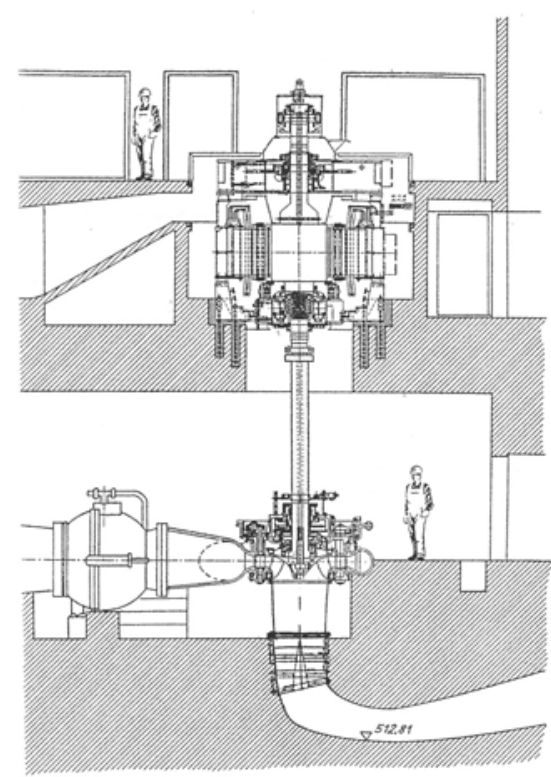

Abb. 4: Kraftwerk Kamering Turbinenwirkungsgrad

wegen des Fehlens eines Speichers als Laufkraftwerk einzusetzen.

Bis zum Erwerb der Anlage durch die KELAG im Jahre 1997 erfolgte der Betrieb des Kraftwerkes von der durchgehend - zwei Mann am Tag und ein Mann in der Nacht - besetzten Schaltwarte aus. Die Maschinen waren nicht automatisiert, mussten manuell gesteuert und auch reguliert werden und wurden für die Eigenstromerzeugung einer Produktionsstätte eingesetzt.

\subsection{Durchgeführte Maßnahmen}

Mit der Kraftwerksübernahme war es ein vordringliches Ziel der KELAG, umgehend und kostengünstig Voraussetzungen für einen wärterlosen Betrieb des Kraftwerkes zu schaffen. Dazu wurde vom betriebseigenen Personal in einem ersten Schritt eine Reihe von Umbau- und Ergänzungsarbeiten durchgeführt. Diese umfassten im Wesentlichen die

- Installation eines Pegelreglers für die wahlweise Ansteuerung einer Francisoder einer Peltonturbine, mit der Aufgabe, die dem jeweiligen Zufluss entsprechende Leistungseinstellung vorzunehmen, bzw. diese Maschine im Bedarfsfall auch automatisch abzustellen. Weitere Maschinen wurden mit fixer Lasteinstellung gefahren.

- Errichtung von Schnellschlusseinrichtungen bei den mechanischen Turbinenreglern der Peltonturbinen;

- Ausstattung der Handantriebe bei den Einlaufschiebern der Peltonturbinen 


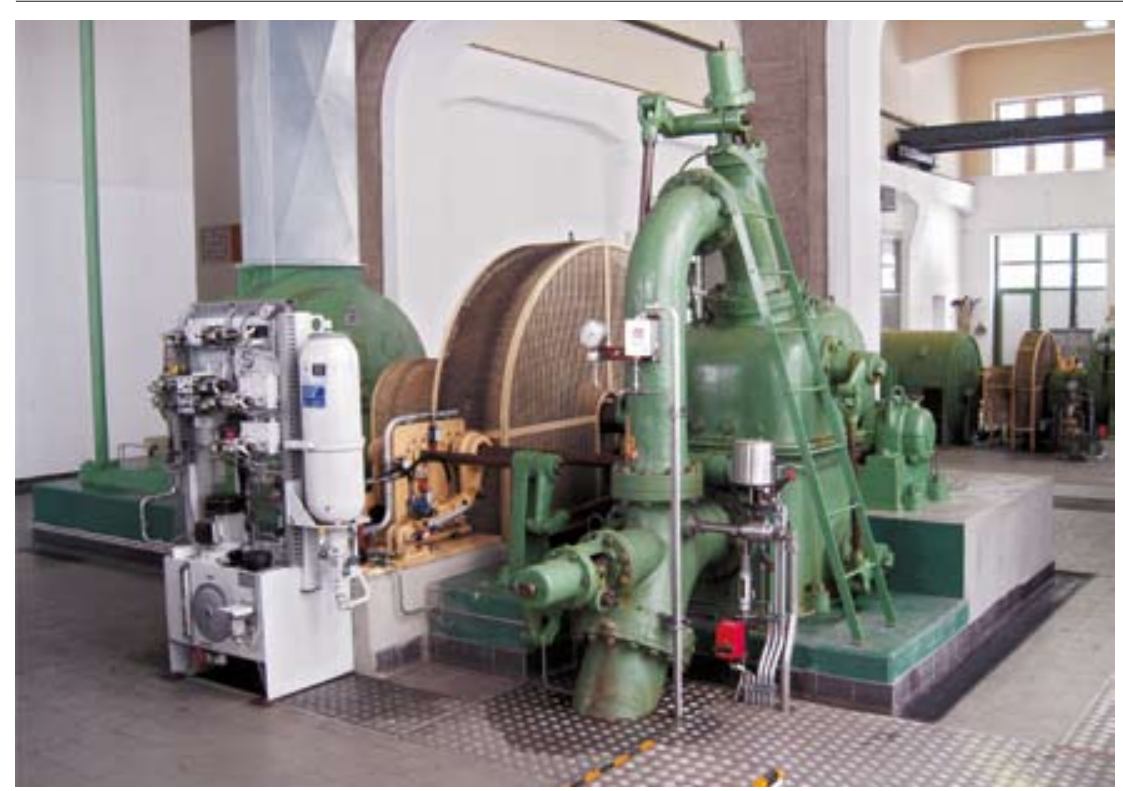

Abb. 5: Peltonturbine nach dem ersten Automatisierungsschritt

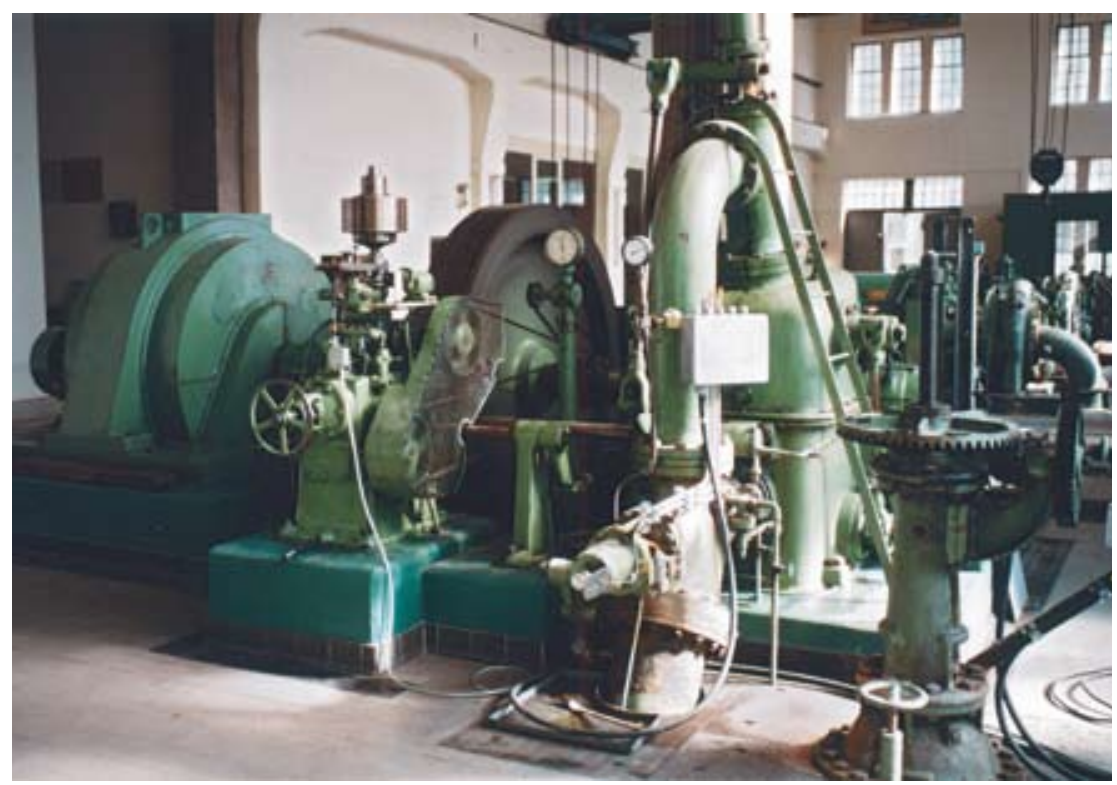

Abb. 6: Peltonturbine nach der Revitalisierung

mit Elektromotoren zur Gewährung einer gesicherten Stillsetzung;

- Einbau zusätzlicher Sensoren bei den Maschinen zwecks Steuerung bzw. Fernüberwachung;

- Einbau einer Gefahrmeldeanlage und Erfassung der wichtigsten Zustandsund Gefahrmeldungen und deren Fernübertragung in die Energieleitzentrale der KELAG nach Klagenfurt;

- Umbau von zwei Schaltzellen, um die galvanische Trennung der Anlagenbereiche des Vorbesitzers mit dem Netz der KELAG zu erreichen.

Die ohne Fremdleistungen getätigten Umbaumaßnahmen beanspruchten einen Zeitraum von zwei Monaten und gestatteten forthin einen wärterlosen Betrieb, der in dieser Form eineinhalb Jahre andauern sollte. Anlagenkontrollen und Lastkorrekturen bei den Maschinen waren nur noch in mehrtägigen Intervallen notwendig.

Um die nahezu 80 Jahre alte Kraftwerksanlage (Peltonmaschinensätze 60 Jahre und Francismaschinensätze 30 Jahre) auf KELAG-Standard zu bringen wurde im Jahre 1999 mit umfangreichen Erneuerungs-, Automatisierungs- und Revitalisierungsmaßnahen begonnen.

Im Einzelnen wurden folgende Maßnahmen getätigt:
- Errichtung einer Kraftwerkleittechnik mit Visualisierung und Protokollierung für den vollautomatischen und wirkungsgradoptimierten Betrieb aller vier Maschinen

- Erneuerung der Erregungseinrichtungen sowie der übrigen Kraftwerkselektrik

- Generalüberholung der Maschinensätze

- Erneuerung der Laufräder bei den Peltonturbinen

- Erneuerung der Keil-Einlaufschieber gegen Kugelhähne bei den Peltonturbinen

- Bestückung der für den Automatikbetrieb erforderlichen Armaturen und Sensoren

- Tausch der mechanischen Turbinenregler gegen hydraulische Reguliereinrichtungen und Hydraulikaggregate

- Überholung und Sanierung von Maschinenteilen

- Neukonservierung

- Druckrohrleitung

- Teilerneuerung der genieteten Stahl-Druckrohrleitung (GFK, erdverlegt)

- Erneuerung der VerteilrohrleitungsAbzweige zu den Peltonturbinen

- Erneuerung der Rohr-Auflagesättel

- Neukonservierung

- Erneuerung von Einlaufrechen und Rechenreinigungsmaschine sowie mehrerer Schütze des Triebwasserweges

- Erneuerung der Krafthaus-Dacheindeckung

\subsection{Fazit}

Durch die angeführten Sanierungs- und Modernisierungsmaßnahmen konnte das Kraftwerk dem Stand der Technik angepasst und die Betriebsführung deutlich im Sinne einer Effizienzsteigerung - verbessert werden.

\section{Kraftwerk Freibach -}

Wiederinbetriebnahme der Speicherpumpe

\subsection{Kurzvorstellung und Historie des Speicherkraftwerkes Freibach}

Das Kraftwerk Freibach liegt etwa $17 \mathrm{~km}$ südöstlich von Klagenfurt im Gemeindegebiet Gallizien.

Mit der Errichtung des Kraftwerkes wurde das Einzugsgebiet der Talflanken im Bergdreieck Hochobir, Koschuta und Freiberg in den Karawanken erschlossen (siehe Abbildung 7: Lageplan Kraftwerk Freibach). 

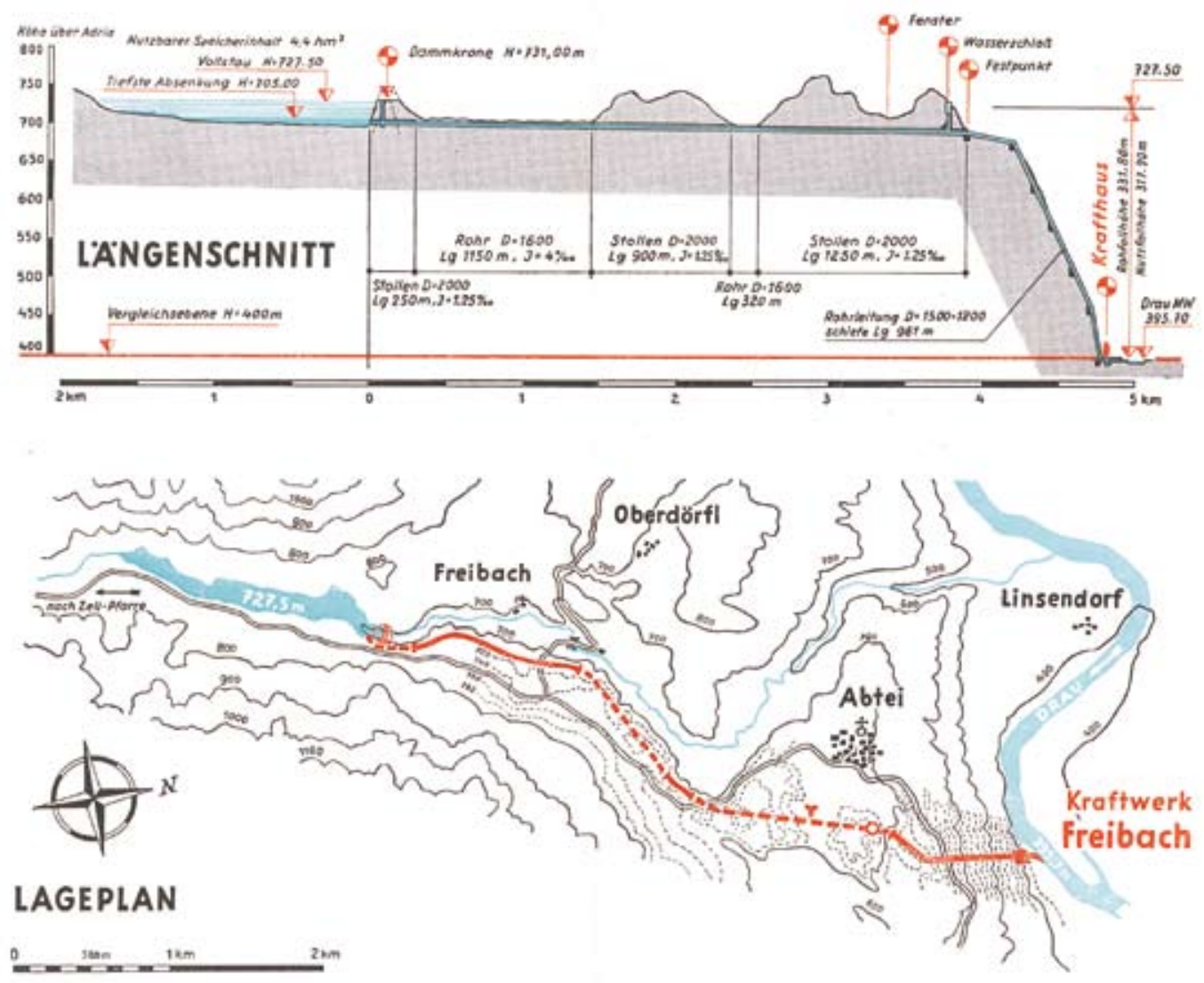

Abb. 7: Lageplan Kraftwerk Freibach

Mit dem Bau der Kraftwerksanlage wurde im Jahr 1957 begonnen. Die maschinelle Ausstattung des Krafthauses erfolgte in zwei Schritten. Der Turbinenmaschinensatz wurde im Jahr 1958 nach nur 1,5 Jahren Bauzeit in Betrieb genommen. Im Jahr 1962 folgte dann auch die Inbetriebsetzung der Speicherpumpe.

Wie man aus dem zeitlichen Verlauf erkennen kann, wurden beim Kraftwerk Freibach zwei getrennte Maschinensätze für Turbinen- bzw. Pumpbetrieb eingebaut. Dies ist mit den unterschiedlichen Anforderungen bezüglich der Ausbauleis$\operatorname{tung}\left(\mathrm{P}_{\mathrm{A}_{-} \text {Turbine }}=16,4 \mathrm{MW}, \mathrm{P}_{\mathrm{A} \_ \text {Pumpe }}=4,7 \mathrm{MW}\right)$ zu erklären.

In Intervallen von 10 Jahren erfolgten an der Speicherpumpe Großrevisionen bei denen sich immer wieder Schwächen bei den Lagern bzw. bei den Wellendichtungen zeigten.

Seit dem Jahr 1969 wird die Speicherpumpe auch als Turbine betrieben.

Im Jahr 1976 begann dann der Bau des Draukraftwerkes Annabrücke. Im Zuge dieser Baumaßnahmen erfolgte im Bereich des Pumpeneinlaufes Kraftwerk Freibach eine Eintiefung der Drau um ca.
$5 \mathrm{~m}$. Damit konnte der Speicherpumpe Freibach das zum Pumpen erforderliche Triebwasser nicht mehr zugeführt werden. Da damals aus wirtschaftlicher Sicht ein Pumpbetrieb im Kraftwerk Freibach nicht erforderlich schien, verzichtete man auf bauliche Maßnahmen um die Pumpe weiterhin mit Triebwasser versorgen zu können. Die Pumpe diente seit diesem Zeitpunkt nur noch als „Notturbine“, als Ersatz für die eigentliche Turbine im Revisionsfall.

\subsubsection{Technische Daten der Speicherpumpe}

Bei der Speicherpumpe handelt es sich um eine einflutige, dreistufige Pumpe mit folgenden technischen Daten:

Förderstrom Q:

$1,16-1,35 \mathrm{~m}^{3} / \mathrm{s}$

Manometrische Förderhöhe HMan: 333-304 m

Max. Wellenleistung PA:

4.700 Kilowatt

Drehzahl n:

745 Upm

Erforderlicher Vordruck HS:

$1,9 \mathrm{~m}$
Der Antriebsmotor ist als Asynchronmotor mit folgenden Daten ausgeführt:

Motorleistung PM:

5.000 Kilowatt

Antriebsdrehzahl nM:

$745 \mathrm{Upm}$

Als druckseitiges Absperrorgan ist ein Eckringschieber installiert, saugseitig sind als Absperrorgane Dammbalken vorgesehen:

Nennweite DN:

$550 \mathrm{~mm}$

Durchflussmenge Q:

$1,16-1,35 \mathrm{~m}^{3} / \mathrm{s}$

Nenndruck PN:

38 bar

Steuerung:

Triebwasser

Da sich im liberalisiertem Strommarkt die Pumpspeicherung wieder als wirtschaftlich erwiesen hat, entschloss sich die KELAG im Jahr 2004 entsprechende bauliche und maschinelle Maßnahmen zu tätigen, um die Speicherpumpe wieder als Pumpe betreiben $\mathrm{zu}$ können. 


\subsection{Notwendige Maßnahmen}

In erster Linie war die sichere Versorgung der Speicherpumpe mit Triebwasser mit dem notwendigen Vordruck von 1,9 m, bezogen auf die erste Stufe der Pumpe, zu planen.

Die Pumpe wurde so geplant und eingebaut, dass das Triebwasser direkt aus dem Unterwasserkanal entnommen werden konnte. Bevor das Kraftwerk Annabrücke mit der damit verbundenen Eintiefung der Drau im Bereich des Zulaufkanals von ca. 5 Metern errichtet wurde, war der Unterwasserkanal immer genügend eingestaut, um die Pumpe mit Triebwasser zu versorgen. Folglich mussten Möglichkeiten ausgearbeitet werden, wie man den Zulaufkanal mit ausreichend Wasser für den Pumpbetrieb füllen kann.

Nach den Vorprüfungen und Machbarkeitsstudien, haben sich zwei Möglichkeiten zur Füllung des Zulaufkanales herauskristallisiert:

- Die erste Möglichkeit: Errichtung einer Vorpumpstation, bestehend aus einem Pumpenschacht mit entsprechendem Einlaufbauwerk, situiert am Ende des bestehenden Unterwasserkanals und ausgestattet mit entsprechenden Pumpen, die zumindest einen Förderstrom von $1,4 \mathrm{~m}^{3} / \mathrm{s}$ von der Drau in den durch den Pumpenschacht abgeschlossen Unterwasserkanal - entspricht in diesem Fall einem kleinen Ausgleichsbecken - fördern.

- Die zweite Möglichkeit: Entnahme des benötigten Triebwassers direkt aus dem Stauraum Annabrücke und Einleitung dieses in den zur Drau hin abgeschlossenen Unterwasserkanal. Dies hätte entweder durch eine Rohrleitung, die durch die bestehende Staumauer mittels entsprechenden Kernbohrungen verlegt worden wäre, oder durch ein naturnahes, freies Gerinne um die Staumauer erfolgen können.

Nach Gesprächen mit dem Betreiber des Kraftwerkes Annabrücke und ersten Kostenermittlungen hat sich gezeigt, dass sich nur die Errichtung einer "Vorpumpstation" wirtschaftlich sinnvoll verwirklichen lässt.

Weiters waren Reparaturen, Adaptierungen und Erneuerungen an der eigentlichen Pumpe und am Eckringschieber notwendig. Außerdem musste die gesamte Maschinensteuerung mit dem mechanischen und elektrischen Maschinenschutz so erneuert werden, dass ein wärterloser Betrieb mit Steuerung aus der
Ferne (Energieleitzentrale in Klagenfurt) möglich wurde. Dies erreichte man durch den Einbau eines modernen Maschinenleitsystems.

\subsection{Vorpumpstation}

Baulich und maschinell musste bei der Errichtung der Vorpumpstation darauf geachtet werden, dass die Pumpe auch weiterhin als "Notturbine“ zu Verfügung stehen konnte und somit der Unterwasserkanal in beide Richtungen durchströmt wird.

Konstruktiv wurde die Vorpumpstation so gestaltet, dass in Verlängerung des Unterwasserkanals ein lotrechter Fallschacht betoniert wurde, der auch gleichzeitig den Kanalabschluss -die notwen- dige Überströmkante- bildet, welcher mit dem ebenfalls ausbetonierten Zulauf zur Drau offen verbunden wurde. Neben dem Fallschacht wurde ein zweiter Schacht errichtet, in dem die Pumpen untergebracht werden konnten.

Um den Betrieb der Vorpumpen nicht durch Schwemmgut zu gefährden, wurde der Zulauf aus der Drau mit einem Feinrechen (Neigung $60^{\circ}$, Stababstand $40 \mathrm{~mm}$ ) und der Fallschacht mit einem geneigten Grobrechen versehen. Die rechteckig ausgeführte Verbindung zwischen dem Fallschacht und dem Pumpenschacht kann durch einen Gleitschütz dicht verschlossen werden, sodass Arbeiten an den Vorpumpen auch ohne dem Setzen eines Dammbalkens im Zulauf möglich sind.

Als wirtschaftlichste Lösung für die

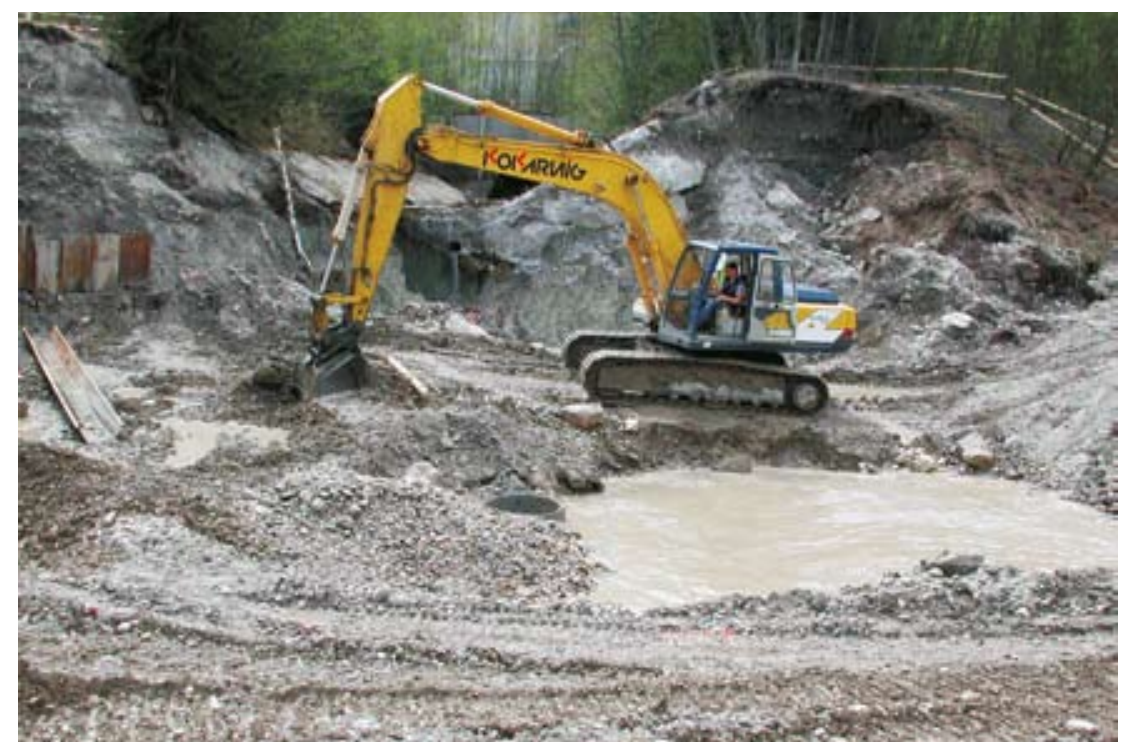

Abb. 8: Erschließung der Baugrube/Vorpumpstation in Betrieb

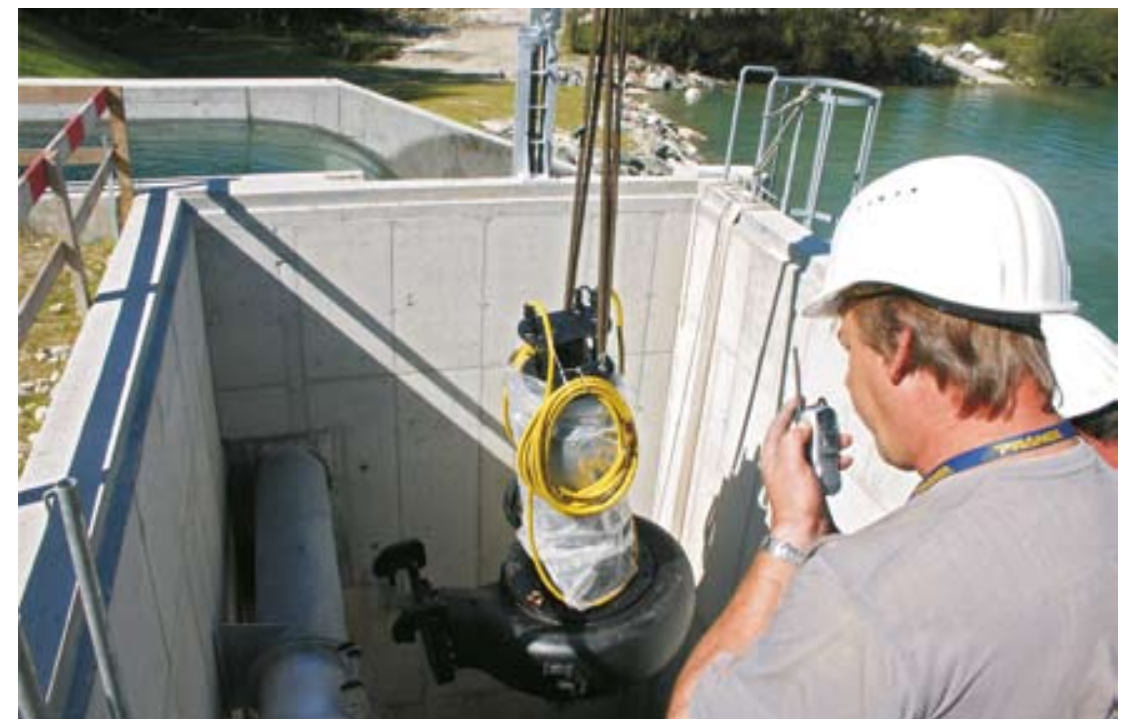

Abb. 9: Montage der Tauchmotorpumpen 


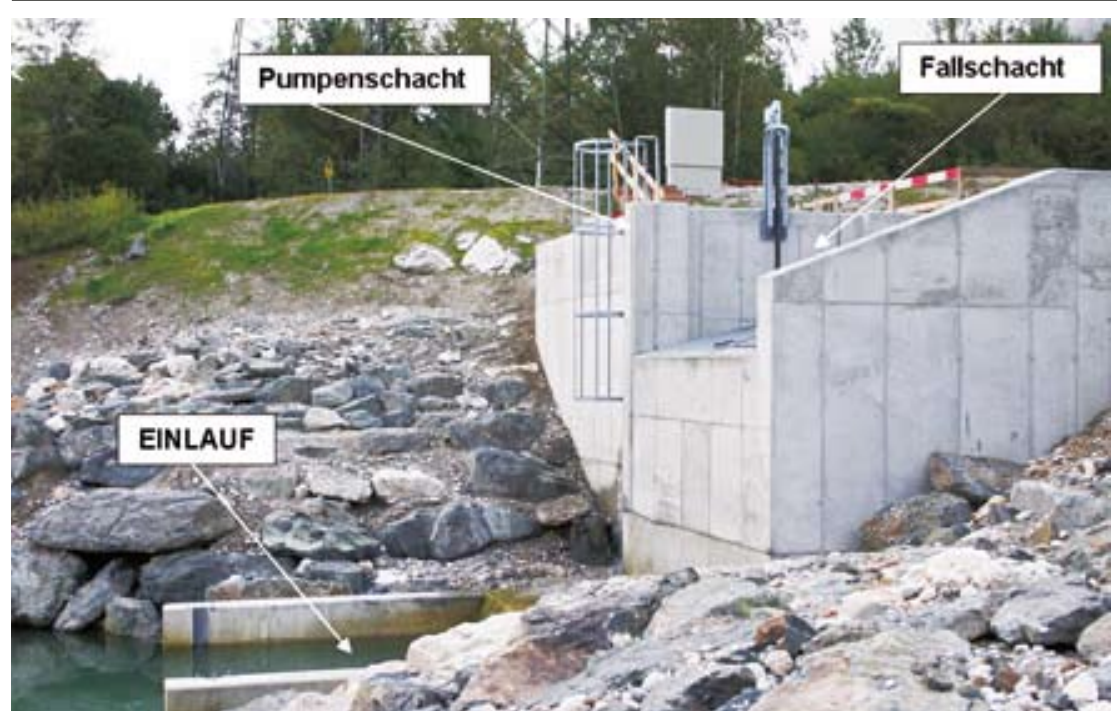

Abb. 10: fertiggestelltes Bauwerk

Ausführung der Vorpumpen haben sich zwei Standard-Tauchmotorpumpen der Fa. WILO ergeben. Diese Pumpen kommen aus der Abwassertechnik und sind entsprechend robust und für längeren Dauerbetrieb ausgelegt.

Der Betriebspunkt der Pumpen liegt bei einer Förderhöhe von 6,8 $\mathrm{m}$ und einer Fördermenge von $750 \mathrm{l} / \mathrm{s}$. Somit sind ausreichende Reserven in der Fördermenge der Vorpumpen gegenüber der Fördermenge der Speicherpumpe vorhanden. Weiters weisen diese Tauchmotorpumpen einen recht guten Wirkungsgrad von ca. $85 \%$ auf. Die Motorenantriebsleistung beträgt 95 Kilowatt. Angefahren werden die Pumpenmotoren über einen Stern-Dreieck-Anlauf.

Für jede Pumpe wurde eine separate Rohrleitung montiert, um die Gefahr von Instabilitäten beim Zusammenschluss zweier Pumpenleitungen zu verhindern. Die Abbildungen 8, 9 und 10 zeigen das Baugeschehen, die Montage der Tauchmotorpumpen und das fertige Vorpumpenbauwerk.

\subsection{Maßnahmen an der Speicherpumpe und Maschinensteuerung}

Um einen problemlosen automatischen, ferngesteuerten und wärterlosen Betrieb der Speicherpumpe gewährleisten zu können, wurden alle Geber und Überwachungseinrichtungen des mechanischen Maschinenschutzes erneuert. Damit sind alle Druck- und Temperaturgeber, sowie Durchflussüberwachungseinrichtungen gemeint.

Weiters wurden die beiden Wellen- dichtungen der Speicherpumpe erneuert und diverse Verschleißteile nachbearbei-

Der Eckringschieber wurde ausgebaut und in der Werkstätte der Kraftwerksgruppe Fragant generalüberholt. Ebenfalls total erneuert wurde dabei die Steuerung des Eckringschiebers.

Die ursprüngliche Pumpenautomatik konnte den neuen Betriebserfordernissen nicht genügen und wurde vollkommendurcheineneue Maschinenleittechnik der Type Symatic S7 ersetzt.

Besonders zu erwähnen ist, dass diese Arbeiten, sowohl Planungs-, Programmierungs- als auch Montagearbeit durch KELAG-Personal durchgeführt wurden.

\subsection{Fazit}

Der im Herbst 2004 begonnene Bau der Vorpumpstation und Umbau an der bestehenden Speicherpumpe und Pumpenautomatik wurde im Sommer 2005 erfolg-

Alle Planungsleistungen, Instandsetzungsmaßnahmen und Reparaturarbeiten sowie die Errichtung aller erforderlichen Gewerke bis auf die Tauchmotorpumpen und die Bauleistungen wurden durch KELAG-Personal erbracht.

Seit Sommer 2005 laufen die Vorpumpen problemlos und versorgen die Speicherpumpe ausreichend mit Triebwasser.

Die Speicherpumpe ist, seit sie wieder in Betrieb gesetzt wurde, ca. $9000 \mathrm{Be}$ triebsstunden im Pumpbetrieb eingesetzt gewesen. Zum Vergleich dazu brachte es die Speicherpumpe vor der Eintiefung der Drau auf gesamt 2400 Betriebsstunden. tet bzw. neu angeschafft. reich abgeschlossen.
Probleme oder Störungen, die einen längeren Stillstand der Speicherpumpe nach sich gezogen hätten, sind bis dato noch nicht aufgetreten. Alle Systeme funktionieren nach Plan.

Die große Anzahl der Betriebsstunden zeigt auch, dass der Einsatz der Speicherpumpe wirtschaftlich ist und sich die getroffenen Maßnahmen bewährt haben.

\section{Kraftwerk Bärental IV}

\subsection{Allgemeines}

Das Kraftwerk Bärental IV wurde im Jahre 1988 zum Zwecke der Brauchwasserversorgung für das Bären-Batteriewerk von der Fa. Jungfer errichtet. Es befindet sich im Kärntner Rosental, an der Aufschließungsstrasse in das Bärental.

Das Kraftwerk IV bezieht sein Triebwasser bei einer Bruttofallhöhe von hBrutto $=182 \mathrm{~m}$ aus einer $4,7 \mathrm{~km}$ langen Druckrohrleitung. Das abgearbeitete Triebwasser wird in ein ca. $20.000 \mathrm{~m}^{3}$ fassendes Speicherbecken abgegeben. Die erzeugte elektrische Energie wird über einen Transformator und eine $5 \mathrm{kV}$-Kabelverbindung in das KELAG-Schaltwerk Feistritz eingespeist.

Im Jahre 1999 wurde das Kraftwerk Bärental IV gemeinsam mit 3 weiteren Kleinwasserkraftwerken im Bärental von der KLEAG übernommen. $\mathrm{Zu}$ diesem Zeitpunkt wurde das Kraftwerk aufgrund einer mangelhaften Automatisierung mit geringer Auslastung betrieben. Der vorhandene Maschinensatz war nicht optimal auf die hydraulischen Gegebenheiten angepasst und lief mit schlechtem Wirkungsgrad.

In diesem Zustand war ein wirtschaftlicher Betrieb der Anlage nicht gegeben. Die KELAG beschloss daher, die Kraftwerksanlage in solchem Ausmaß zu revitalisieren, dass eine Erhöhung des Regelarbeitsvermögens von mehr als $50 \%$ erreicht wird.

\subsection{Zustand der Anlage vor der Revitalisierung}

\subsubsection{Wasserfassung}

Die Wehranlage besteht aus einer festen Betonschwelle und einem Grundablassfeld. Der Einlauf des Triebwassers erfolgt über einen Grobrechen, in einen offenen Entkieser mit Spülmöglichkeit. Anschließend durchströmt das Triebwasser einen Feinrechen und zwei offene Entsander- 


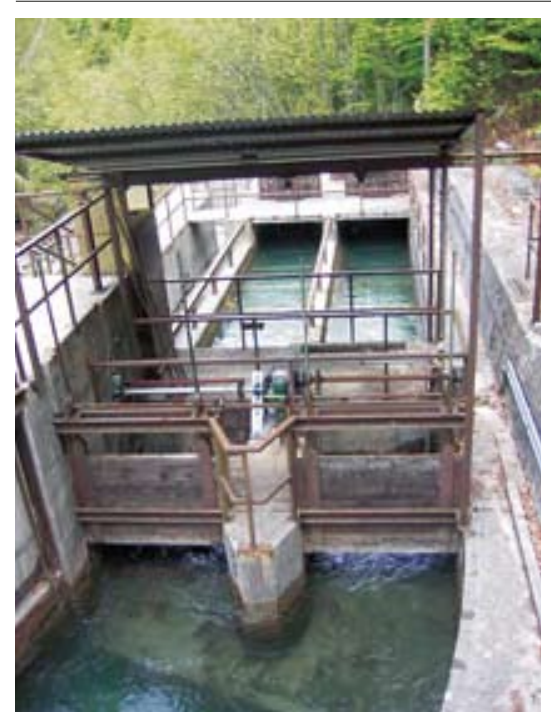

Abb. 11: Wasserfassung

kammern, die ebenfalls mit einer Spülmöglichkeit ausgestattet sind. Im Anschluss an die Entsanderkammer ist der Einlauf in die Druckrohrleitung mit vorgelagertem, fernauslösbarem Fallschütz situiert.

Alle Spülschütze waren mit Handbetrieb ausgestattet. Die Steuerung der Rechenreinigungsmaschinen erfolgte durch händisches Einschalten vor Ort.

\subsubsection{Turbine}

Installiert war eine 2-düsige Peltonturbine. Die Regelung erfolgte mittels eines ölhydraulischen Doppelreglers.

Die Turbine wurde seinerzeit gebraucht zugekauft (Baujahr 1945). Sie war nicht optimal an die hydraulischen Gegebenheiten angepasst. Die Düsenführungskreuze, ausgeführt mit sechs Stegen, waren häufig verlegt.

Die Nennleistung der Turbine wurde mit 768 Kilowatt angegeben. Im Betrieb konnte jedoch nur eine maximale Leistung von 450 Kilowatt erreicht werden. Dabei staute sich das Triebwasser in der Turbine und quoll in weiterer Folge aus den Labyrinthen. Der Turbinenwirkungsgrad betrug unter den gegebenen Betriebsbedingungen nach Berechnungen der KELAG maximal $55 \%$.

\subsubsection{Generator}

Ein Asynchrongenerator, Baujahr 1987, war direkt (ohne Schwungmasse) an die Turbine gekoppelt. Der Generator war für die Turbine überdimensioniert und wurde somit nur im Teillastbereich, bei schlechtem Wirkungsgrad gefahren.

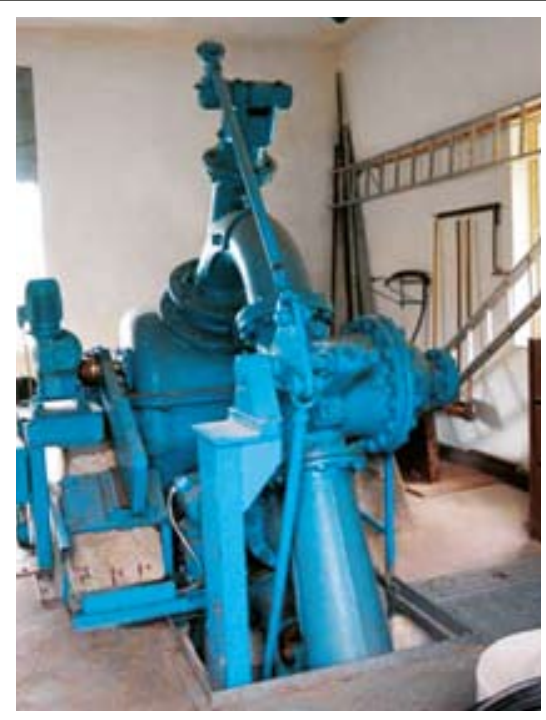

Abb. 12: alte Peltonturbine

\subsubsection{Maschinenautomatik,} Kraftwerksleittechnik

Eine Maschinenautomatik war nicht vorhanden. Das Anfahren, Stillsetzen und Synchronisieren der Maschine wurde vor Ort manuell durchgeführt. Die Betätigung der Regeleinrichtungen erfolgte per Hand.

Die Maschinenleistung wurde nicht selbsttätig dem Wasserdargebot angepasst, daher wurde die Anlage auch nicht automatisch bei optimalem Wirkungsgrad betrieben. Im unbesetzten Betrieb kam es bei Überwasser zu Erzeugungsausfällen.

\subsection{Revitalisierungsmaßnahmen}

\subsubsection{Allgemeines}

Für einen optimalen, wirtschaftlichen Betrieb der Anlage wurden Investitionsmaßnahmen mit dem Ziel einer Verbesserung des Gesamtwirkungsgrades und einer Erhöhung der Betriebsstunden durchgeführt.

Das Kraftwerk wurde in einem Ausmaß revitalisiert, dass eine Erhöhung des Regelarbeitsvermögens von mehr als 50\% erreicht werden konnte.

Die durchgeführten Maßnahmen führten zu keiner Erhöhung der Engpassleistung.

\subsubsection{Maßnahmen in Einzelnen}

\subsubsection{Wasserfassung}

Zur Verbesserung der Betriebsführung wurden alle Schützantriebe (Grundablass-, Einlauf-, Spül- und Absperrschütz) automatisiert.

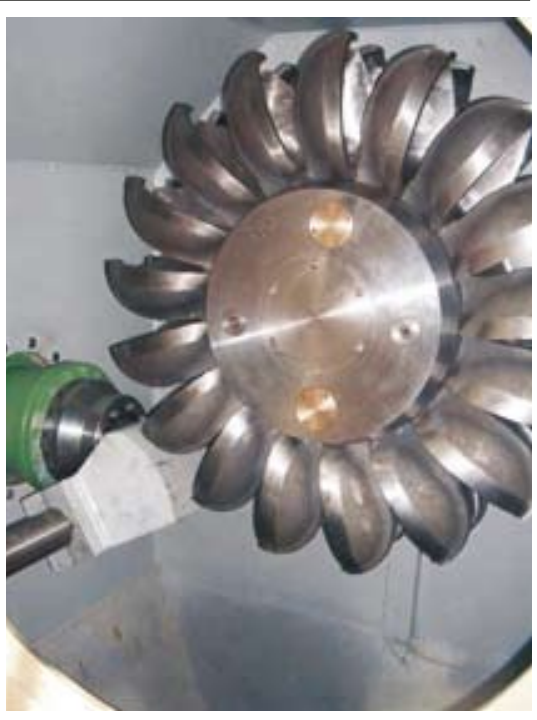

Abb. 13: neue Peltonturbine/Laufrad

Die Rechenreinigungsmaschine des Feinrechens wurde generalüberholt und automatisiert. Durch diese Maßnahmen ist jetzt ein funktionierender Wassereinzug gewährleistet, längere Steh- und Ausfallszeiten werden vermieden.

\subsubsection{Turbine}

Die vorhandene Turbine wurde durch eine neue, optimal an die hydraulischen Gegebenheiten angepasste, Turbine ersetzt.

Technische Daten der Turbine: 1-düsige Pelton-Turbine Fabrikat: EFG Turbinenbau Ausbauwassermenge Q: $370 \mathrm{l} / \mathrm{s}$ Fallhöhe H: $170 \mathrm{~m}$

Nennleistung P: 0,460 MW Nenndrehzahl n: $750 \mathrm{rpm}$ Nennwirkungsgrad: $89,7 \%$

\subsubsection{Generator}

Der vorhandene Asynchrongenerator wurde durch einen an die Turbine angepassten Synchrongenerator ersetzt. Das Turbinenlaufrad ist fliegend auf der Generatorwelle montiert. Die Auswahl der optimalen Generatorspannung ( $400 \mathrm{~V}$ ) führte zu einer weiteren Verbesserung des Gesamtwirkungsgrades.

Die Netzrückwirkung wurde durch den Einsatz eines Synchrongenerators ebenfalls maßgeblich verringert.

Technische Daten des Generators:

Fabrikat: AEM Dessau

Type: SE 450 M8

Nennspannung: 400/231 V 


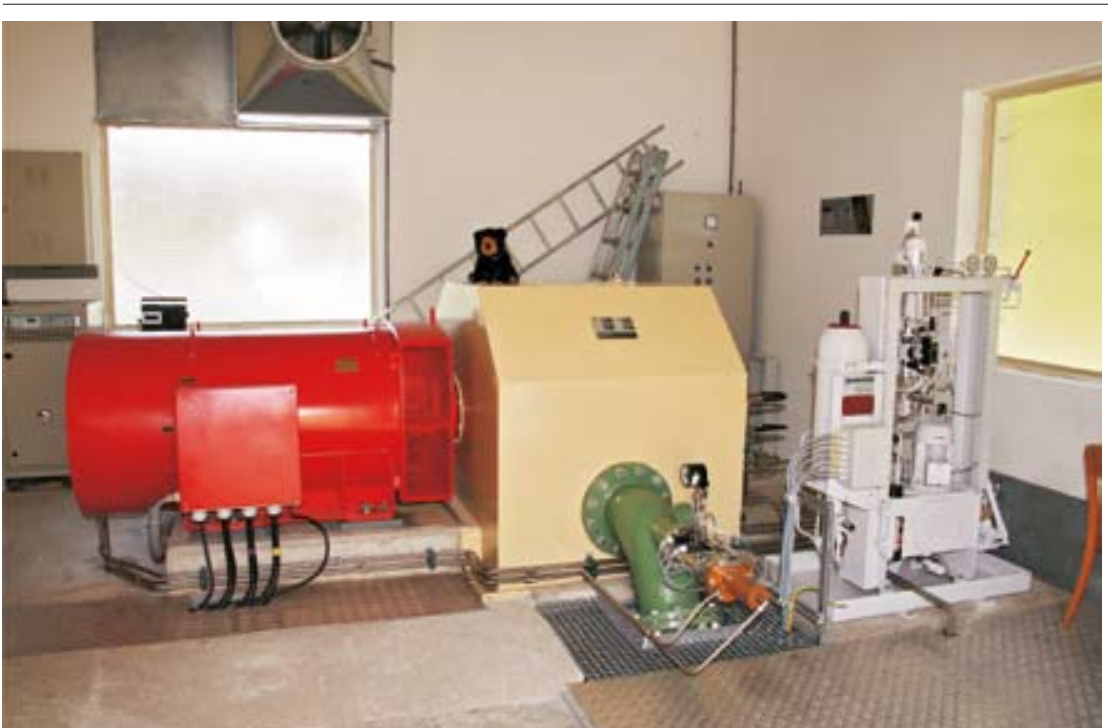

Abb. 14: neuer Synchrongenerator mit neuer Peltonturbine

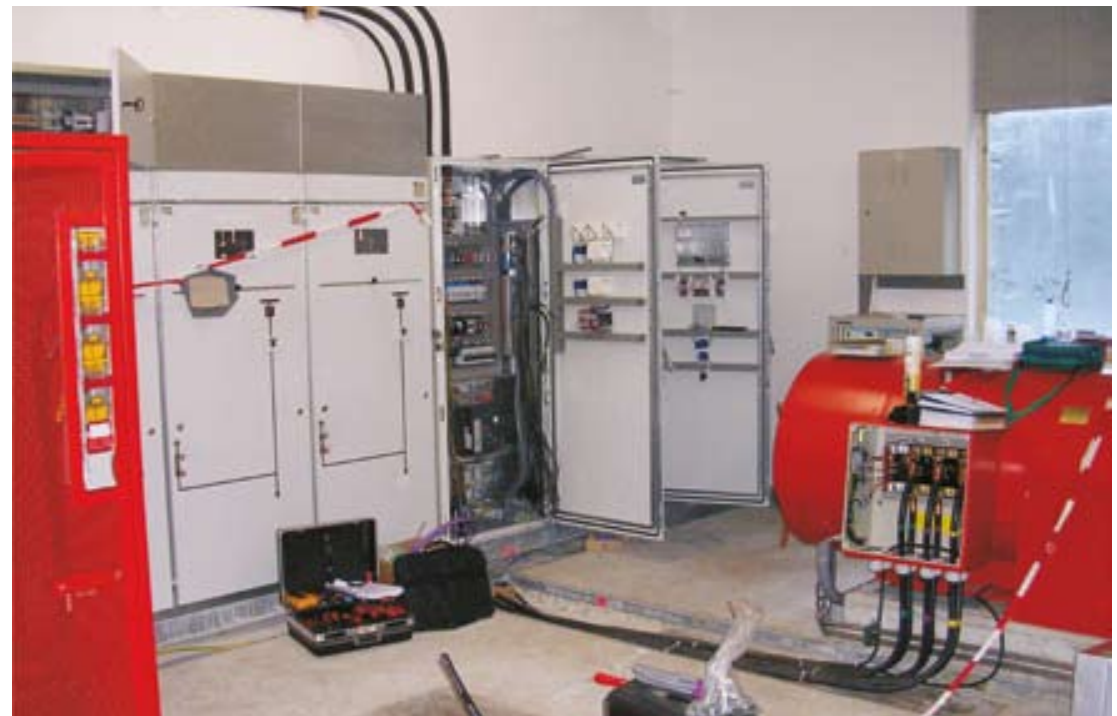

Abb. 15: neue Maschinenautomatik mit Schaltanlage der Anlage ermöglicht. Entsprechend dem Wasserdargebot wird die Anlage bei optimalem Wirkungsgrad betrieben. Durch die neue in Digitaltechnik ausgeführte Maschinenautomatik werden Ausfallsund Stehzeiten verkürzt und damit verbundene Erzeugungsverluste verringert. Durch geringere Wartungsstillstände wurde eine weitere Betriebsstundenerhöhung erreicht.

\subsection{Fazit}

Durch die beschriebenen Maßnahmen wurde für gleiche Betriebsbedingungen eine Erhöhung des theoretischen Regelarbeitsvermögens von weit über $50 \%$ erreicht. Die geplanten Maßnahmen führten zu keiner Erhöhung der Engpassleistung.

\section{Korrespondenz:}

Prok. Dipl.-Ing. Gerald BERGER

KELAG - Kärntner

Elektrizitäts-Aktiengesellschaft

Erzeugung

Arnulfplatz 2, 9020 Klagenfurt, Österreich

Telefon: +43 463/525-1470, FAX: +43 463/525-1561

E-Mail: gerald.berger@KELAG.at

\section{Ing. Johann KLAUSNER}

KELAG - Kärntner

Elektrizitäts-Aktiengesellschaft

Kraftwerksgruppe Fragant
Außerfragant 72, 9831 Flattach, Österreich

Telefon: +43 4785/8108-5201, FAX: +43 463/525-95520 E-Mail: johann.klausner@kelag.at

Dipl.-Ing. Stefan LEITNER

KELAG - Kärntner

Elektrizitäts-Aktiengesellschaft

Erzeugung/Kraftwerksplanung

Arnulfplatz 2, 9020 Klagenfurt, Österreic

Telefon: +43 463/525-1542, FAX: +43 463/525-156

E-Mail: stefan.leitner@kelag.at

Dipl.-Ing. Ingo PREISS

KELAG - Kärntne

Elektrizitäts-Aktiengesellschaft

Erzeugung/Kraftwerksplanung

Arnulfplatz 2, 9020 Klagenfurt, Österreich

Telefon: +43 463/525-1488, FAX: +43 463/525-951488 E-Mail: ingo.preiss@kelag.at
Nennscheinleistung: 0,535 MVA

Leistungsfaktor: 0,9

Nenndrehzahl n: $750 \mathrm{U} / \mathrm{min}$

max. Wirkungsgrad: $96,03 \%$ (bei $0,8 x S n$ )

\subsubsection{Regeleinrichtung}

Der mechanische Turbinenregler wurde durch einen digitalen Regler, integriert in die Maschinenleittechnik, ersetzt. Die Verstellung der Düsen und des Leitapparates erfolgt ölhydraulisch.

Für einen pegelgeregelten Betrieb der Anlage wurden im Einlaufbauwerk Sonden zur Pegelerfassung installiert.

\subsubsection{Maschinenautomatik, Kraftwerksleittechnik}

Durch die neuen Regeleinrichtungen wurde ein automatischer Betrieb (Anfahr-, Abstell- und Wiederzuschaltautomatik) 